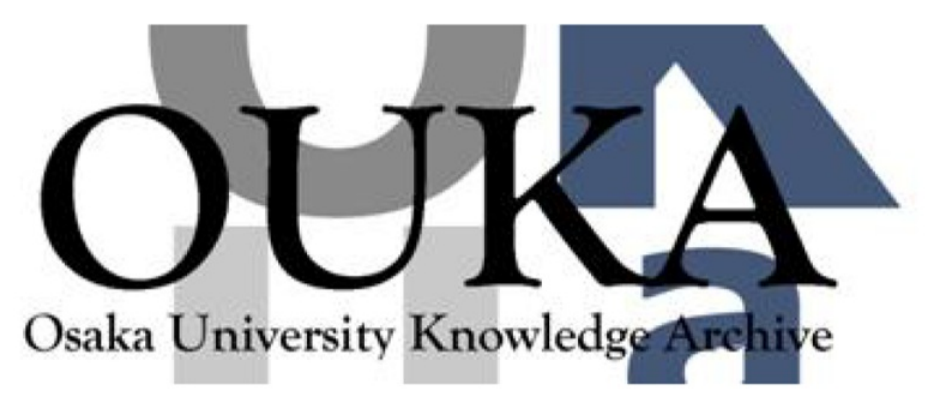

\begin{tabular}{|c|l|}
\hline Title & $\begin{array}{l}\text { Detection of abnormal myocardial deformation } \\
\text { during acute myocardial ischemia using three- } \\
\text { dimensional speckle tracking echocardiography }\end{array}$ \\
\hline Author(s) & $\begin{array}{l}\text { Hioki, Ayana; Asanuma, Toshihiko; Masuda, } \\
\text { Kasumi et al. }\end{array}$ \\
\hline Citation & Journal of Echocardiography. 18(1) p. 57-p. 66 \\
\hline Issue Date & $2020-03-01$ \\
\hline oaire:version & AM \\
\hline URL & https://hdl. handle. net/11094/85578 \\
\hline rights & \\
\hline Note & \\
\hline
\end{tabular}

Osaka University Knowledge Archive : OUKA

https://ir. Library. osaka-u. ac. jp/

Osaka University 


\section{Detection of Abnormal Myocardial Deformation During Acute Myocardial Ischemia Using Three-Dimensional Speckle Tracking Echocardiography}

Ayana Hioki, Toshihiko Asanuma, Kasumi Masuda, Daisuke Sakurai, Satoshi Nakatani

Division of Functional Diagnostics, Department of Health Sciences, Osaka University Graduate School of Medicine, Suita, Osaka, Japan

Concise title: Detection of Abnormal Deformation by 3D Echocardiography

Contribution of each author:

Ayana Hioki study design, analysis and interpretation of data, drafting of the manuscript

Toshihiko Asanuma study design, analysis and interpretation of data, drafting of the manuscript

Kasumi Masuda analysis and interpretation of data, revising of the manuscript

Daisuke Sakurai interpretation of data, revising of the manuscript

Satoshi Nakatani interpretation of data, revising of the manuscript

Reprint request and correspondence:

Dr. Toshihiko Asanuma

Division of Functional Diagnostics, Department of Health Sciences, Osaka University Graduate School of Medicine, 1-7 Yamadaoka, Suita, Osaka, 565-0871, Japan

E-mail: toshi@sahs.med.osaka-u.ac.jp 


\begin{abstract}
Background: Three-dimensional (3D) speckle tracking echocardiography can simultaneously evaluate circumferential, longitudinal, and radial strain without being affected by through-plane motion. Moreover, the assessment of area change ratio may allow measuring regional myocardial deformation more accurately. We investigated the changes in each deformation parameter during acute coronary flow reduction, and evaluated whether the spatial extent of the abnormal values in each deformation parameter corresponded to that of the perfusion abnormality.

Methods: In 10 dogs, myocardial strains of three directions and area change ratio were analyzed at baseline and during three different ischemic conditions. The peak systolic value and the post-systolic index (PSI) were measured in both the ischemic and normal segments. The function abnormality, derived from the deformation parameter, and the perfusion abnormality, derived from Evans blue staining, were evaluated in each segment during complete occlusion and the concordance rate between both abnormalities was calculated.

Results: In all deformation parameters, the peak systolic value tended to gradually decrease and the PSI tended to gradually increase with the severity of flow reduction in the ischemic segment. Especially in area change ratio, significant changes were observed in both the peak systolic value and the PSI during occlusion compared to baseline. The concordance rate was the highest in the PSI assessed by area change ratio.
\end{abstract}

Conclusions: Among 3D myocardial deformation parameters, area change ratio demonstrated better detectability of acute coronary flow reduction than conventional strain components. Area change ratio may be a useful parameter for detecting acute ischemia by $3 \mathrm{D}$ speckle tracking echocardiography.

Keywords: myocardial ischemia, myocardial strain, post-systolic shortening, three-dimensional echocardiography. 


\section{Introduction}

Two-dimensional (2D) speckle tracking echocardiography allows the quantitative assessment of myocardial deformation, and facilitates the detection of subtle myocardial motion, such as post-systolic shortening (PSS) [1,2]. Post-systolic shortening is a sensitive marker of myocardial ischemia and the analysis of PSS improves the diagnostic accuracy for ischemia compared to conventional systolic wall thickening [3-6]. However, through-plane motion of the left ventricle (LV) in the 2D images affects speckle tracking data and may cause inaccurate evaluation of the myocardial strain parameters $[7,8]$.

In contrast, three-dimensional (3D) speckle tracking echocardiography can simultaneously evaluate circumferential, longitudinal, and radial strain without being affected by through-plane motion [9-11]. Moreover, area change ratio, which is measured as percentage change in a segmental endocardial surface area, has been reported to provide a more accurate measurement of regional myocardial deformation compared to one-dimensional myocardial strain [12]. Although 3D speckle tracking echocardiography appears to be useful to assess regional deformation for diagnosis of myocardial ischemia, its low spatial and temporal resolution may hamper detecting the subtle deformation abnormality during ischemia.

In this study, we investigated the changes in each parameter derived from 3D speckle tracking echocardiography to assess myocardial deformation in different levels of acute coronary flow reduction. We also evaluated whether the spatial extent of the abnormal values in each deformation parameter corresponded to that of the perfusion abnormality.

\section{Methods}

\section{Animal preparation}

All animal studies were approved by the animal experimentation committee and performed in accordance with guidelines for the care and use of laboratory animals at our institution (Osaka University). A total of 10 open-chest female mongrel dogs (weight: $14.6 \pm 0.7$ 
$\mathrm{kg}$ ) were used. Dogs were anesthetized using intravenous pentobarbital sodium $(35 \mathrm{mg} / \mathrm{kg})$, intubated, and ventilated using a respirator pump. Oxygen saturation was monitored with a pulse oximeter and maintained within normal ranges. Anesthesia with pentobarbital sodium was maintained throughout the experiment $(6.3-7.1 \mathrm{mg} / \mathrm{kg} / \mathrm{h})$. Buprenorphine hydrochloride (4 $\mu \mathrm{g} / \mathrm{kg}$ ) was administered intramuscularly as an analgesic. The electrocardiogram was monitored continuously, and the LV pressure was measured with a 5-F micromanometer (Millar Instruments, Houston, TX, USA). The heart was suspended in a pericardial cradle through a left lateral thoracotomy in the fifth intercostal space. The proximal portion of the left circumflex coronary artery (LCx) was dissected free from the surrounding tissues, and a snare occluder was placed in this position. A perivascular ultrasonic flow probe (Transonic Systems, Ithaca, NY, USA) was set at the distal site of the occluder and connected to a digital flowmeter.

\section{Echocardiography}

Three-dimensional echocardiographic datasets were acquired by an Aplio Artida ultrasound system with a PST-25PX matrix array transducer (Toshiba, Otawara, Tochigi, Japan). Because of the left lateral thoracotomy in the upper intercostal space, the transducer was fixed above the LV lateral wall through a water bath as a standoff. After confirming that the apex of the LV was included in the cross-sectional images, full-volume datasets were acquired from six continuous cardiac cycles using the electrocardiogram-gating technique and a pyramidal image $\left(57.2 \times 62.5^{\circ}\right)$ was reconstructed. In acquiring datasets, respiration was transiently stopped to avoid stitching artifacts. The transmitting and receiving frequency was set at 2.0 and $4.0 \mathrm{MHz}$, respectively. The volume rate was set at the highest rate that we could select unless the lateral resolution was visually deteriorated $(34-36 \mathrm{~Hz})$. A microphone for the phonocardiogram was placed nearby the base of the aorta. The timing of aortic valve closure (AVC) was determined from the aortic component of the second heart sound derived from the phonocardiogram [13]. End-diastole was defined at the R-wave of the electrocardiogram and end-systole was defined at the timing of AVC. The end-systolic frame was determined as the closest one from the timing of 
AVC.

\section{Experimental protocol}

We examined three different ischemic conditions (two levels of flow-limiting stenosis and a brief complete occlusion) of the LCx. After acquiring datasets at baseline, the LCx was gradually narrowed with the occluder in the following order: $20 \%-50 \%$ flow reduction (stenosis 1), $50 \%-80 \%$ flow reduction (stenosis 2 ), and 100\% flow reduction (complete occlusion). In stenosis 1 and 2, datasets were acquired at least 1 minute after controlling the flow reduction and keeping the stable systemic and coronary hemodynamics. In complete occlusion, datasets were acquired 1 minute after the LCx occlusion. The occluder was released after each ischemic condition, and the following stenosis or occlusion was controlled after the full recovery of the LCx flow and the hemodynamics to the baseline level.

LV pressure was recorded at the same time as the acquisition of the $3 \mathrm{D}$ echocardiographic datasets. LV systolic pressure, LV end-diastolic pressure, and maximum and minimum time derivatives of the $\mathrm{LV}$ pressure $\left(\mathrm{dP} / \mathrm{dt}_{\max }, \mathrm{dP} / \mathrm{dt}_{\min }\right)$ were averaged from five consecutive cardiac cycles.

We used Evans blue dye into the left atrium after the LCx ligation at the end of the protocol. After dye injection and euthanasia, the heart was excised and cut from the apex to the base into eight transverse slabs.

\section{Data analysis}

The 3D speckle tracking analysis was performed with offline software (Toshiba, Otawara, Tochigi, Japan) (Figure 1). The algorithm of speckle tracking in this software was described in previous articles [9-11]. In the 4- and 2-chamber views cut out from the 3D datasets, the endocardial borders, excluding papillary muscles, were visually identified, and contours were manually traced on an end-diastolic frame. The epicardial borders were determined by setting an even width of myocardium. The endocardial and epicardial borders were then automatically tracked for all frames in the clip. Tracking accuracy of both borders was visually confirmed in 4- 
and 2-chamber views and three levels of short-axis images, and adjusted if needed. After the confirmation, three myocardial strain parameters (circumferential strain, longitudinal strain, and radial strain) and area change ratio were analyzed in 16 segments of the LV. Area change ratio, which reflects the product of circumferential and longitudinal strains but is not the same, was calculated from the average change of the unit areas defined by four tracking points [12]. Using this software, values of the radial strain were calculated from the endocardial and epicardial borders, and those of the circumferential strain, longitudinal strain, and area change ratio were calculated from only the endocardial border. The reference point (i.e., zero strain) was set at end-diastole.

To investigate the changes in each myocardial deformation parameter derived from 3D speckle tracking echocardiography during acute coronary flow reduction, the peak systolic value in each parameter was measured in the ischemic segment (mid-posterior segment) and in the opposite normal segment (mid-anteroseptal segment). When PSS was observed in the strain or area change ratio curve, the amplitude of PSS was measured. The post-systolic index (PSI) was calculated from a ratio, (amplitude of PSS) / (maximum amplitude during the cardiac cycle), as a parameter of PSS (Figure 1) [5]. When PSS was not observed, the PSI represents zero in this definition.

Next, to evaluate whether the spatial extent of the abnormal segments in each deformation parameter corresponded to that of the abnormal segments in myocardial perfusion during complete occlusion, a mean \pm standard deviation (SD) in each deformation parameter was analyzed per segment from the data at baseline in 10 dogs. We then assessed whether each segment had normal function (within \pm 2 SDs of mean at baseline) or abnormal function (beyond \pm 2 SDs of mean at baseline) during complete occlusion. We also assessed whether each segment had normal perfusion (stained myocardium) or abnormal perfusion (non-stained myocardium) with Evans blue staining during LCx ligation. The segments that included the border of Evans blue staining were excluded from the analysis because the simple binary assessment was 
conducted. When the segment had "normal function and normal perfusion" or "abnormal function and abnormal perfusion", it was assessed as concordant. In contrast, when the segment had "normal function and abnormal perfusion" or "abnormal function and normal perfusion", it was assessed as discordant. The concordance rate was evaluated as (the number of concordant segments) / (the number of analyzed segments).

\section{Statistical analysis}

Data are expressed as a mean $\pm \mathrm{SD}$. Multiple comparisons in each hemodynamic parameter were performed by one way analysis of variance with the Tukey-Kramer post hoc test. Because of heterogeneity of variance, multiple comparisons in each myocardial deformation parameter were performed by the Games-Howell post hoc test in the ischemic and normal segments separately. Interobserver and intraobserver reliability for each parameter was determined by intra-class correlation coefficients (ICCs). One full-volume image clip per dog, in which one cardiac cycle was included, was randomly selected from the baseline, stenosis, and occlusion conditions. To determine the interobserver ICCs, the analyses of a total of 10 clips were repeated by a second observer who was blinded to the measurement performed by the first observer. To determine the intraobserver ICCs, the analyses of the same 10 clips were repeated two or more weeks later by the same observer. In the repeated measures, the values in the ischemic segment (mid-posterior segment) were compared. Statistical significance was set at $\mathrm{p}<$ 0.05 .

\section{Results}

\section{Hemodynamics}

By changes of the electrocardiographic wave during acute ischemia, electrocardiogram-gated acquisition of 3D images failed during complete occlusion in one dog, and failed during stenosis 2 and complete occlusion in another dog. Since these data were excluded from the analysis, the analyzed data were as follows: 10 (baseline), 10 (stenosis 1), 9 
(stenosis 2), and 8 (complete occlusion).

LCx flow gradually decreased in accordance with the definition of three different ischemic conditions. LV end-diastolic pressure tended to increase and the absolute value of $\mathrm{dP} / \mathrm{dt}_{\min }$ tended to decrease during occlusion relative to baseline (Table 1 ).

\section{Myocardial deformation curves derived from 3D speckle tracking echocardiography}

The myocardial deformation curves of circumferential strain, longitudinal strain, radial strain, and area change ratio at baseline and three different ischemic conditions in the ischemic and normal segments of a dog are shown in Figure 2. In this dog, the absolute peak systolic values in the ischemic segment decreased with the severity of flow reduction in all parameters, except that the longitudinal peak systolic value decreased more during stenosis 1 than during stenosis 2. PSS in the ischemic segment tended to become larger with the severity of flow reduction. In contrast, the absolute peak systolic values in the normal segment clearly increased during occlusion as a result of hyperkinetic motion.

\section{Peak systolic value and PSI in each deformation parameter}

In all analyzed data, the absolute peak systolic values tended to gradually decrease with the severity of flow reduction in the ischemic segment in all deformation parameters. In circumferential strain and area change ratio, the peak systolic values during occlusion significantly decreased compared to those at baseline. On the other hand, the peak systolic values in the normal segment tended to increase during the flow reduction procedure (Figure 3).

The PSI tended to gradually increase with the severity of flow reduction in the ischemic segment in all parameters. In longitudinal strain and area change ratio, the PSI during occlusion significantly increased compared to those at baseline (Figure 4).

\section{Concordance between each deformation parameter and perfusion}

In each deformation parameter, a mean $\pm \mathrm{SD}$ at baseline could be obtained per segment. Table 2 shows the peak systolic value and the PSI derived from area change ratio at baseline.

In four of the eight dogs in which data acquisition during occlusion succeeded, a clear 
discrimination between stained and non-stained myocardium with Evans blue staining was difficult. Therefore, the remaining four dogs were used for the analysis of concordance between function and perfusion. The concordance rate between function and perfusion was finally evaluated in 47 segments after excluding the borderline segments of Evans blue staining.

The concordance rate of the PSI tended to be higher than that of the peak systolic value in each deformation parameter except radial strain. The concordance rate of area change ratio tended to be higher than those of the other strain parameters in the peak systolic value, and also tended to be higher in the PSI. Therefore, the PSI assessed by area change ratio demonstrated the highest concordance rate (89\%) (Figure 5).

\section{Interobserver and intraobserver ICCs}

In the peak systolic value, the interobserver and intraobserver ICCs $(95 \%$ confidence intervals) were $0.66(0.14-0.90)$ and $0.92(0.73-0.98)$ for circumferential strain, $0.79(0.38-0.94)$ and $0.97(0.91-0.99)$ for longitudinal strain, $0.95(0.81-0.99)$ and $0.81(0.44-0.95)$ for radial strain, and $0.75(0.28-0.93)$ and $0.94(0.78-0.98)$ for area change ratio.

In the PSI, the interobserver and intraobserver ICCs were $0.88(0.60-0.97)$ and 0.96 $(0.85-0.99)$ for circumferential strain, $0.73(0.25-0.92)$ and $0.95(0.81-0.99)$ for longitudinal strain, $0.97(0.86-0.99)$ and $0.75(0.29-0.93)$ for radial strain, and $0.74(0.28-0.93)$ and 0.98 (0.92-0.99) for area change ratio.

\section{Discussion}

In this study, the changes in myocardial deformation parameters derived from 3D speckle tracking echocardiography were investigated in different levels of acute coronary flow reduction. In all parameters tested, the peak systolic value tended to gradually decrease and the PSI tended to gradually increase in the ischemic segment with the severity of flow reduction. Especially in area change ratio, significant changes were observed in both the peak systolic value and the PSI during occlusion compared to baseline. The concordance rate between function and perfusion 
was the highest in the PSI assessed by area change ratio.

\section{D speckle tracking echocardiography}

Although speckle tracking analysis by $2 \mathrm{D}$ echocardiography is a useful method for quantifying regional myocardial deformation, it has several limitations including through-plane motion. To overcome these limitations, 3D speckle tracking echocardiography was made available in the clinical settings. Theoretically, it is expected that accurate strains can be measured by the 3D analysis because of the lack of through-plane motion [7,8]. Moreover, data acquisition of $3 \mathrm{D}$ echocardiography is faster than that of $2 \mathrm{D}$ echocardiography since the acquisition of multiple cross-sectional views is not necessary [14]. Given that in patients with acute myocardial ischemia it is important to acquire the data in a short time, the efficiency of quantitative assessment by $3 \mathrm{D}$ echocardiography would be desirable for detecting acute ischemia.

On the other hand, there are also disadvantages to 3D speckle tracking echocardiography. For example, spatial and temporal resolution of the 3D images, which is inferior to that of 2D echocardiography, is an unavoidable limitation [15]. Since the accuracy of speckle tracking depends on the image quality, lower spatial and temporal resolution may influence the evaluation of myocardial deformation and the diagnosis of acute ischemia. To date, however, detectability of acute ischemia in each 3D speckle tracking parameter has not been fully investigated.

\section{Detection of acute myocardial ischemia by 3D speckle tracking echocardiography}

In our 3D study, the peak systolic values tended to decrease and the PSI tended to increase in the ischemic segment with the severity of flow reduction in all deformation parameters. In our previous 2D study, peak systolic strain tended to decrease and the PSI significantly increased with flow reduction in radial strain derived from tissue Doppler echocardiography [16]. Moen et al. measured circumferential, longitudinal, and radial strain by 2D tissue Doppler and speckle tracking analyses in pigs with four different levels of coronary 
flow reduction and showed that peak systolic strain significantly decreased and the PSS significantly became larger with the severity of flow reduction in each deformation parameter [17]. The data generated in the present study are consistent with these results.

In the results of Moen et al., peak systolic strain and PSS changed significantly during more than $50 \%$ flow reduction [17]. In contrast, the 3D parameters in the present study tended to change in a similar fashion (stenosis 2), but the changes were not significant. This discrepancy appears to be due to lower spatial and temporal resolution of 3D echocardiography, which would cause inaccurate 3D speckle tracking. This is especially true for lower temporal resolution, which can underestimate the peak value in each $3 \mathrm{D}$ parameter. The frame rate in $2 \mathrm{D}$ speckle tracking of Moen et al. could be set at over $100 \mathrm{~Hz}$, but the volume rate in our 3D speckle tracking was approximately $35 \mathrm{~Hz}$. Low volume rates are permissible for 3D speckle tracking echocardiography due to no through-plane motion [15]; however, because the heart rates were high in our dogs (approximately $160 / \mathrm{min}$ ), our volume rate was relatively low to the heart rate (approximately 13 frames /cardiac cycle), meaning that the true peak strain might be underestimated.

Area change ratio, which is derived from 3D speckle tracking echocardiography, is measured on the basis of the deformation of the regional area in the endocardial surface mesh. Seo et al. demonstrated the accuracy of 3D speckle tracking echocardiography for assessing area change ratio by comparing it to sonomicrometry and demonstrated that the peak value in area change ratio decreased significantly during coronary occlusion [12]. Their results suggested that area change ratio could provide a more accurate measurement of regional myocardial deformation compared to that of myocardial strain because the combined data of two directional (i.e., circumferential and longitudinal) deformations could decrease the tracking error.

In the present study, only area change ratio was significantly changed in both the peak systolic value and the PSI during occlusion compared to baseline. One of the reasons of the improved diagnostic ability in area change ratio may be due to the decrease in the tracking error. 
Although lower spatial and temporal resolution of the present 3D echocardiography decreases the tracking accuracy, the analysis of area change ratio appears to minimize this limitation. It has also been reported that area strain, which is almost the same as area change ratio, can disclose wall motion abnormality in peri-ischemic segments [18].

In the analysis of concordance between function and perfusion, the PSI assessed by area change ratio revealed the highest concordance rate. As shown in Figure 3 and 4, the discrimination between baseline and occlusion was best in the PSI assessed by area change ratio because the standard deviation at baseline was extremely small. This better discrimination appears to explain why the concordance rate of the PSI assessed by area change ratio was the highest.

\section{Clinical implications}

PSS is considered a sign of myocardial ischemia because it occurs in the myocardium with contractile dysfunction through an imbalance of tension between the damaged and surrounding normal myocardium [6]. However, it has been thought that PSS is difficult to be detected by 3D echocardiography because the temporal resolution may not be sufficient to detect subtle myocardial deformation. In fact, few previous reports have demonstrated the usefulness of PSS to diagnose myocardial ischemia by 3D echocardiography. However, our results suggest that PSS is detectable during acute ischemia by the present 3D speckle tracking echocardiography. The assessment of PSS derived from area change ratio may improve the diagnostic accuracy of myocardial ischemia by 3D echocardiography.

In 3D speckle tracking echocardiography, decreased peak systolic values are sometimes observed even in the normal segments. The additional use of the PSI may help discriminating between physiological variation of the peak systolic value and pathological dysfunction more accurately because PSS is myocardial deformation that reflects relative deterioration of active contraction [6].

\section{Study limitations}


In dogs, the area perfused through the LCx is larger than that through the left anterior descending artery (LAD), and the flow of the LCx is greater than that of the LAD. We chose only the LCx territory in which stable flow reduction can be created easily. However, it is still unclear whether the same results are obtained in other territories.

In the present study, the visualization of apical views was impossible since the left lateral thoracotomy was performed through the upper intercostal space. Therefore, we had to fix the transducer to the lateral wall in order to acquire the 3D images (the positional relationship between the LV long-axis and the transducer was perpendicular). The lateral resolution of our 3D images was approximately half of the axial resolution. Although 3D images of the whole heart could be acquired (because a dog's heart is relatively small), the spatial resolution in each 3D direction with this approach should be different from that obtained using the apical approach; this difference in approach might influence the strain values in each direction. However, in area change ratio, this influence seems to be minimized because the advantage and disadvantage of spatial resolution in the circumferential and longitudinal directions are offset by each other.

Low temporal resolution might affect the determination of the end-systolic frame; indeed, the detection of small PSS, which is expected to occur during stenosis, appeared to be difficult in cooperation with the low spatial resolution of 3D. However, in clinical cases, in which the heart rate is lower in almost patients, area change ratio may be able to detect ischemia induced by stenosis, although further investigations are necessary.

Because of the experiment in anesthetized open-chest dogs, the values at baseline were lower than those in healthy subjects [19]. Note that cutoff values for assessing abnormal function in the present study cannot be used in the clinical setting.

\section{Conclusions}

In all 3D speckle tracking parameters, the peak systolic value tended to gradually decrease and the PSI tended to gradually increase in the ischemic segment with the severity of flow reduction. Among the deformation parameters, only area change ratio significantly changed 
in both the peak systolic value and the PSI during occlusion. The concordance rate between function and perfusion was the highest in the PSI assessed by area change ratio. Thus, area change ratio may be a useful parameter for detecting acute ischemia by 3D speckle tracking echocardiography. 


\title{
Acknowledgments
}

This research was supported by a Japan Society for the Promotion of Science Grant-in-Aid for Scientific Research (JSPS KAKENHI grant number JP24300181).

\section{Disclosure of conflict of interest}

\author{
Ayana Hioki no disclosure \\ Toshihiko Asanuma no disclosure \\ Kasumi Masuda no disclosure \\ Daisuke Sakurai no disclosure \\ Satoshi Nakatani a research grant from Canon Medical Systems
}

\begin{abstract}
Animal studies
Animal studies: All institutional and national guidelines for the care and use of laboratory animals were followed.
\end{abstract}




\section{References}

1. Amundsen BH, Helle-Valle T, Edvardsen T, et al. Noninvasive myocardial strain measurement by speckle tracking echocardiography: validation against sonomicrometry and tagged magnetic resonance imaging. J Am Coll Cardiol. 2006;47:789-93.

2. Klaeboe LG, Edvardsen T. Echocardiographic assessment of left ventricular systolic function. J Echocardiogr. 2019;17:10-6.

3. Pislaru C, Belohlavek M, Bae RY, et al. Regional asynchrony during acute myocardial ischemia quantified by ultrasound strain rate imaging. J Am Coll Cardiol. 2001;37:1141-8.

4. Voigt JU, Exner B, Schmiedehausen K, et al. Strain-rate imaging during dobutamine stress echocardiography provides objective evidence of inducible ischemia. Circulation. 2003;107:2120-6.

5. Asanuma T, Fukuta Y, Masuda K, et al. Assessment of myocardial ischemic memory using speckle tracking echocardiography. JACC Cardiovasc Imaging. 2012;5:1-11.

6. Asanuma T, Nakatani S. Myocardial ischaemia and post-systolic shortening. Heart. 2015;101:509-16.

7. Wu VC, Takeuchi M, Otani K, et al. Effect of through-plane and twisting motion on left ventricular strain calculation: direct comparison between two-dimensional and three-dimensional speckle tracking echocardiography. J Am Soc Echocardiogr. 2013;26:1274-81.

8. Seo Y, Ishizu T, Atsumi A, et al. Three-dimensional speckle tracking echocardiography: A promising tool for cardiac functional analysis. Circ J. 2014;78:1290-301.

9. Kawagishi T. Speckle tracking for assessment of cardiac motion and dyssynchrony. Echocardiography. 2008;25:1167-71.

10. Seo Y, Ishizu T, Enomoto Y, et al. Validation of 3-dimensional speckle tracking imaging to quantify regional myocardial deformation. Circ Cardiovasc Imaging. 2009;2:451-9. 
11. Maffessanti F, Nesser HJ, Weinert L, et al. Quantitative evaluation of regional left ventricular function using three-dimensional speckle tracking echocardiography in patients with and without heart disease. Am J Cardiol. 2009;104:1755-62.

12. Seo Y, Ishizu T, Enomoto Y, et al. Endocardial surface area tracking for assessment of regional LV wall Deformation with 3D speckle tracking imaging. JACC Cardiovasc Imaging. 2011;4:358-65.

13. Masuda K, Asanuma T, Taniguchi A, et al. Assessment of dyssynchronous wall motion during acute myocardial ischemia using velocity vector imaging. JACC Cardiovasc Imaging. $2008 ; 1 ; 210-20$.

14. Altman M, Bergerot C, Aussoleil A, et al. Assessment of left ventricular systolic function by deformation imaging derived from speckle tracking: A comparison between 2D and 3D echo modalities. Eur Heart J Cardiovasc Imaging. 2014;15:316-23.

15. Yodwut C, Weinert L, Klas B, et al. Effects of frame rate on three-dimensional speckle-tracking-based measurements of myocardial deformation. J Am Soc Echocardiogr. 2012;25:978-85.

16. Okuda K, Asanuma T, Hirano T, et al. Impact of the coronary flow reduction at rest on myocardial perfusion and functional indices derived from myocardial contrast and strain echocardiography. J Am Soc Echocardiogr. 2006;19:781-7.

17. Moen CA, Salminen PR, Grong K, et al. Left ventricular strain, rotation, and torsion as markers of acute myocardial ischemia. Am J Physiol Heart Circ Physiol. 2011;300:H2142-54.

18. Ternacle J, Gallet R, Champagne S, et al. Changes in three-dimensional speckle-tracking-derived myocardial strain during percutaneous coronary intervention. J Am Soc Echocardiogr. 2013;26:1444-9.

19. Kleijn SA, Pandian NG, Thomas JD, et al. Normal reference values of left ventricular strain using three-dimensional speckle tracking echocardiography: Results from a multicentre 
study. Eur Heart J Cardiovasc Imaging. 2015;16:410-6. 


\section{Table}

\section{Table 1. Hemodynamics}

\begin{tabular}{|c|c|c|c|c|}
\hline & Baseline & Stenosis 1 & Stenosis 2 & Occlusion \\
\hline $\begin{array}{c}\text { LCx flow } \\
{[\mathrm{mL} / \mathrm{min}]} \\
\text { (Flow reduction) }\end{array}$ & $\begin{array}{c}16 \pm 4 \\
(0 \%)\end{array}$ & $\begin{array}{c}11 \pm 3 * \\
(30.8 \pm 8.1 \%)\end{array}$ & $\begin{array}{c}6 \pm 3 * \dagger \\
(65.1 \pm 9.8 \%)\end{array}$ & $\begin{array}{c}0 \pm 0 * \dagger \dagger \\
(100 \%)\end{array}$ \\
\hline $\begin{array}{c}\text { Heart rate } \\
{[\mathrm{bpm}]}\end{array}$ & $160 \pm 9$ & $160 \pm 10$ & $160 \pm 10$ & $165 \pm 10$ \\
\hline $\begin{array}{c}\text { LVSP } \\
{[\mathrm{mmHg}]}\end{array}$ & $107 \pm 11$ & $106 \pm 13$ & $106 \pm 11$ & $103 \pm 11$ \\
\hline $\begin{array}{l}\text { LVEDP } \\
{[\mathrm{mmHg}]}\end{array}$ & $6 \pm 3$ & $7 \pm 3$ & $7 \pm 3$ & $9 \pm 3$ \\
\hline $\begin{array}{c}\mathrm{dP} / \mathrm{dt}_{\max } \\
{[\mathrm{mmHg} / \mathrm{s}]}\end{array}$ & $1538 \pm 208$ & $1502 \pm 225$ & $1542 \pm 197$ & $1525 \pm 266$ \\
\hline $\begin{array}{c}\mathrm{dP} / \mathrm{dt}_{\min } \\
{[\mathrm{mmHg} / \mathrm{s}]}\end{array}$ & $-1720 \pm 274$ & $-1656 \pm 321$ & $-1605 \pm 546$ & $-1584 \pm 262$ \\
\hline
\end{tabular}

${ }^{*} \mathrm{p}<0.05$ vs. baseline; $\uparrow \mathrm{p}<0.05$ vs. stenosis $1 ; \sharp \mathrm{p}<0.05$ vs. stenosis 2 .

LCx = left circumflex coronary artery; LVSP = left ventricular systolic pressure; LVEDP = left ventricular end-diastolic pressure; $\mathrm{dP} / \mathrm{dt}_{\max }=$ maximum time derivatives of left ventricular pressure; $\mathrm{dP} / \mathrm{dt}_{\min }=$ minimum time derivatives of left ventricular pressure. 
Table 2. Peak systolic value and post-systolic index (PSI) derived from area change ratio at baseline

\begin{tabular}{|c|c|c|c|}
\hline & Basal & Mid & Apical \\
\hline \multicolumn{4}{|c|}{ Peak systolic value (\%) } \\
\hline Anterior & $-11.2 \pm 4.6$ & $-15.0 \pm 4.4$ & $-19.3 \pm 8.7$ \\
\hline Anteroseptal & $-14.2 \pm 3.9$ & $-18.8 \pm 7.5$ & \\
\hline Septal & $-21.2 \pm 5.3$ & $-26.4 \pm 8.3$ & $-22.5 \pm 10.6$ \\
\hline Inferior & $-19.0 \pm 5.3$ & $-24.4 \pm 9.2$ & $-28.1 \pm 12.0$ \\
\hline Posterior & $-14.4 \pm 2.7$ & $-19.2 \pm 8.0$ & \\
\hline Lateral & $-14.2 \pm 3.4$ & $-14.7 \pm 7.3$ & $-24.5 \pm 11.6$ \\
\hline \multicolumn{4}{|l|}{ PSI } \\
\hline Anterior & $0.10 \pm 0.23$ & $0.04 \pm 0.10$ & $0.08 \pm 0.25$ \\
\hline Anteroseptal & $0.00 \pm 0.00$ & $0.04 \pm 0.12$ & \\
\hline Septal & $0.02 \pm 0.04$ & $0.02 \pm 0.06$ & $0.11 \pm 0.27$ \\
\hline Inferior & $0.01 \pm 0.01$ & $0.00 \pm 0.00$ & $0.04 \pm 0.11$ \\
\hline Posterior & $0.06 \pm 0.11$ & $0.00 \pm 0.01$ & \\
\hline Lateral & $0.11 \pm 0.11$ & $0.05 \pm 0.07$ & $0.02 \pm 0.05$ \\
\hline
\end{tabular}

Each value represents a mean \pm standard deviation. 


\section{Figure legends}

\section{Fig. 1 3D speckle tracking echocardiography}

Left: The 4- and 2-chamber views and three levels of short-axis images cut out from 3D echocardiography. Using the 3D speckle tracking analysis, myocardial deformation curves of four myocardial deformation parameters (circumferential strain, longitudinal strain, radial strain, and area change ratio) were analyzed in 16 segments of the left ventricle. Right: The peak systolic value in the area change ratio (A-SYS) and the post-systolic index (PSI), which is a parameter of post-systolic shortening (PSS). The amplitude of PSS (A-PSS) was determined as an absolute difference between the end-systolic value and the peak value (i.e., the minimal value in the area change ratio) after end-systole. The PSI was calculated from a ratio, A-PSS / (maximum amplitude during the cardiac cycle: $\mathrm{A}-\mathrm{MAX}$ ). $\mathrm{AVC}=$ aortic valve closure.

\section{Fig. 2 Myocardial deformation curves derived from 3D speckle tracking}

\section{echocardiography}

Myocardial deformation curves of circumferential strain, longitudinal strain, radial strain, and area change ratio at baseline and three different ischemic conditions in a representative dog. In accordance with the severity of flow reduction, absolute peak systolic values in the ischemic segment decreased and post-systolic shortening (PSS) became larger in all parameters, although the longitudinal peak systolic value decreased more during stenosis 1 than during stenosis 2 . In contrast, absolute peak systolic values in the normal segment clearly increased during occlusion as a result of hyperkinetic motion. $\mathrm{AVC}=$ aortic valve closure.

\section{Fig. 3 Peak systolic value in each deformation parameter}

Absolute peak systolic values tended to gradually decrease with the severity of flow reduction in the ischemic segment in all 3D parameters. In circumferential strain and area change ratio, the peak systolic values during occlusion significantly decreased compared to those at baseline. On 
the other hand, the peak systolic values in the normal segment tended to increase with flow reduction. ${ }^{*} \mathrm{p}<0.05$ vs. baseline.

\section{Fig. 4 Post-systolic index (PSI) in each deformation parameter}

The PSI tended to gradually increase with the severity of flow reduction in the ischemic segment in all 3D parameters. In longitudinal strain and area change ratio, the PSI during occlusion significantly increased compared to that at baseline. ${ }^{*} \mathrm{p}<0.05$ vs. baseline.

\section{Fig. 5 Concordance rate between each deformation parameter and perfusion}

The concordance rate of the post-systolic index (PSI) tended to be higher than that of the peak systolic value in all parameters, with the exception of radial strain, and the concordance rate of area change ratio tended to be higher than those of the other strain parameters. The PSI assessed by area change ratio demonstrated the highest concordance rate. 